\title{
EpiDOL: Epidemic Density Adaptive Data Dissemination Exploiting Opposite Lane in VANETs
}

\author{
Irem Nizamoglu ${ }^{1}$, Sinem Coleri Ergen ${ }^{2}$, and Oznur Ozkasap ${ }^{1}$ \\ 1 Department of Computer Engineering \\ 2 Department of Electrical Engineering \\ Koc University, Turkey \\ \{inizamoglu, sergen, oozkasap\}@ku.edu.tr
}

\begin{abstract}
Vehicular ad-hoc networks (VANETs) aim to increase the safety of passengers by making information available beyond the driver's knowledge. The challenging properties of VANETs such as their dynamic behavior and intermittently connected feature need to be considered when designing a reliable communication protocol in a VANET. In this study, we propose an epidemic and density adaptive protocol for data dissemination in vehicular networks, namely EpiDOL, which utilizes the opposite lane capacity with novel probability functions. We evaluate the performance in terms of end-to-end delay, throughput, overhead and usage ratio of the opposite lane under different vehicular traffic densities via realistic simulations based on SUMO traces in ns-3 simulator. We found out that EpiDOL achieves more than $90 \%$ throughput in low densities, and without any additional load to the network $75 \%$ throughput in high densities. In terms of throughput EpiDOL outperforms the Edge-Aware and DV-CAST protocols $10 \%$ and $40 \%$ respectively.
\end{abstract}

\section{Introduction}

For improving safety of the roads, vehicular ad-hoc networks (VANETs) has become popular both in industry and academia. Efficient usage of vehicular networks has significant potential considering the fact that the amount of traffic accidents is massive. For instance, every year just in the United States almost six million traffic accidents, ten thousands of deaths and millions of injuries occur [1]. Certainly, VANETs are expected to significantly improve the safety of our transportation systems by making information available beyond the drivers' knowledge. In ad-hoc networks, routing protocols are crucial to maintain reliable and efficient communication. Despite this importance, most of the protocols offered for VANET are reinterpretations of the well known mobile ad-hoc network (MANET) routing protocols. However, VANETs behave in fundamentally different ways than the models that predominate in MANET researches. Unlike traditional MANETs, they have high but more predictable mobility models with rapid changes due to high speed and frequent fragmentation in network topology [2]. Geographic position information is available through Global Positioning

T. Bauschert (Ed.): EUNICE 2013, LNCS 8115, pp. 221-232, 2013.

(C) IFIP International Federation for Information Processing 2013 
System (GPS) with no power or hard delay constraints. Therefore, communication methods developed for VANETs must consider various dimensions including delay and reliability requirements, protocol specifications, vehicle mobility, topology characteristics and physical constraints. Thus, instead of using existing protocols proposed for MANETs, design of protocols specifically for VANETs to mitigate its disadvantages and utilize GPS information is needed.

In this study, we propose and develop a novel data dissemination protocol for VANETs. In contrast to prior studies, our protocol EpiDOL uses epidemic routing by using the advantage of opposite lane as relaying with novel probability functions among vehicles. Epidemic technique introduces intelligence into data dissemination by reducing contentions and collisions while not requiring infrastructure support. Our probability functions are simple but effective in providing adaptivity to density. Therefore, the comparison of our approach with state of the art protocols DV-CAST [3] and Edge-Aware [4 in realistic traces, proved the efficiency of EpiDOL in terms of lower delay, higher throughput rates and better utilization of opposite lane relaying. The novelties of this work as following.

- We propose and develop a density adaptive epidemic data dissemination protocol, EpiDOL which uses only limited network information and control flags that indicate packet dissemination direction and vehicles' movement direction.

- In EpiDOL, we propose a new probabilistic approach to utilize opposite lane nodes as relay node to solve disconnected networks problem.

The original contribution is to propose probabilistic density adaptive epidemic data dissemination protocol, EpiDOL. Also, we analyze the dependence of optimal parameters of epidemic routing on the density and the direction of vehicles. In addition, we evaluate the performance of EpiDOL compared with the current routing algorithms with simulations that use realistic traffic traces.

The rest of this paper is organized as follows. Section 2 describes related works. Section 3 discusses the system model. Section 4 describes the details of EpiDOL with performance metrics that we used during the simulations. Section 5 provides scenarios used in simulations and performance criteria. Section 6 presents simulation results and Section 7 concludes the paper.

\section{Related Works}

Distributing Internet on roads [5] or using mobile phones and in-car embedded devices for collecting and processing data [6] are possible applications of VANET. To realize these applications, we have to disseminate the data throughout a network. While thinking ad-hoc networks, the simplest and the most common way of data dissemination is flooding. However, as a result of the redundant broadcasts there may be contentions and collisions in the shared wireless medium. VANET routing protocols mainly deal with two problems, broadcast storm and disconnected networks 4 . When high number of nodes start to disseminate their packets at the same time, it is highly probable that the collisions will occur. The 
loss of data packets due to these collisions are defined as broadcast storm problem. [7] and [8] try to solve this problem in MAC layer level by including some new ideas such as disseminating packets with probability functions. [9] develops a scheme to distribute packets fairly to the network by using the local knowledge. We have further improved these approaches by introducing probability functions adaptive to density. EpiDOL uses epidemic approach with different probability functions succeeded to decrease the packet loss and overhead while increasing the throughput significantly.

According to [10] other challenges in VANET i.e. vehicle movements and driver's behavior cause rapid topology changes and frequent fragmentation on the network. Possible link breakages are predicted using the velocity of the nodes in 11 and 12. We deal these problems by using periodically updated neighborhood info. Additionally the dynamic behaviors of the vehicles and the sparsely connected networks introduce new problems. However it is already shown that Gossip-based (Epidemic) protocols are effective to solve these problems and provide reliable and efficient communication [13].

Reactive and proactive protocols have different behaviors under different traffic regimes [2], they should be robust to different density levels. [14] presents a zone-based forwarding scheme to deal with density problems. At low density networks the disconnected network problem is a severe issue. It can be defined as the case when there are not sufficiently enough nodes for data transmission in the network. It is proposed that by choosing the best packet structure this problem can be solved [11. To deal with this problem, the proposal and successful implementation of the intelligible use of the vehicles in the opposite lane for relaying packets between disconnected networks in the original lane is another novel contribution of this work.

[15] and [16] reinterpret well-known protocols AODV, GPSR and OLSR instead of proposing specialized approaches for VANET. Addition to this different techniques, Edge-aware Epidemic Protocol [3] is the most relevant study to this work. Edge-aware detects edge nodes and assigns high probabilities to these nodes. However, EpiDOL extends this approach with its opposite lane usage and simple but effective probabilistic forwarding technique.

\section{System Model}

We consider an ad-hoc network with randomly distributed vehicles on a multilane bidirectional highway. Each vehicle is equipped with GPS and has the communication capability with 802.11 p protocol [17. We assumed that the nodes are only interested in the packets that are generated by nodes that lead them within less than a certain region of interest (ROI). All packets are generated by a leading node with the same priority and the same dissemination distance. Any packet can be lost due to collisions, however, if a packet is received successfully, then there are no bit errors that lead to the misinformation of the node.

Since there is no central control or clustering mechanism, all nodes in the network act independently. They are only aware of their neighbors' locations 
and directions by periodical updates which will be discussed in Section 4. It is assumed that any further information about the network topology or density are not available to the vehicles.

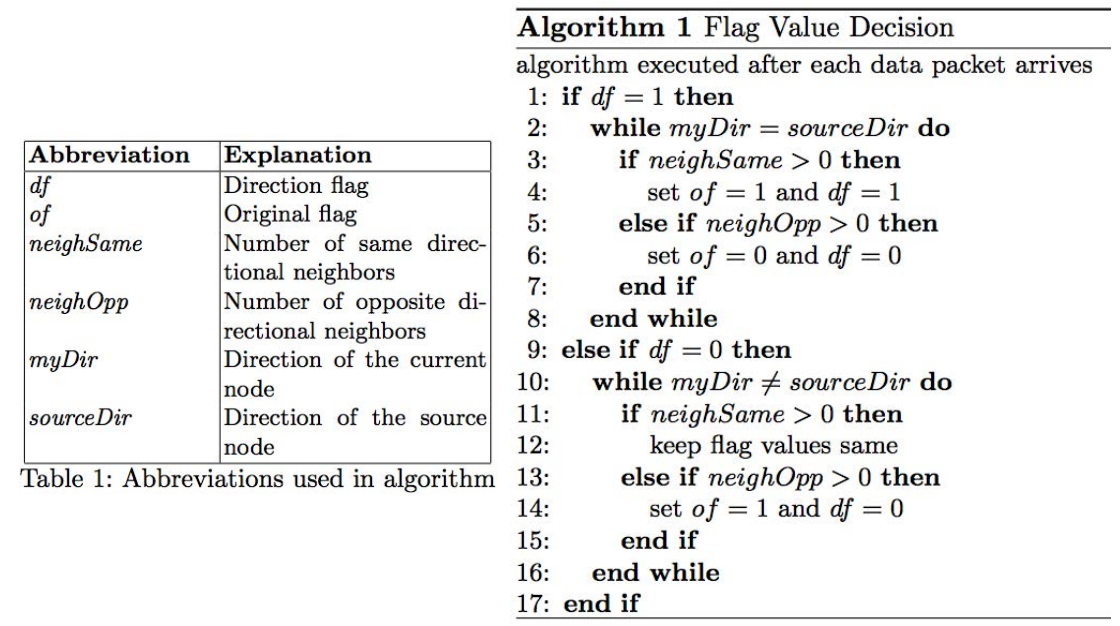

\section{Algorithm Description}

In this section, we describe principles of EpiDOL with its system architecture and probability functions.

\subsection{System Architecture}

In our approach, we aim to create an intelligent packet dissemination system by using flags on application layer. We use two binary flags: of shows the actual dissemination direction of the data packet, and $d f$ shows the vehicles riding direction with respect to the direction of the source node. With the help of the information that we gathered from these flags, the opposite lane is used effectively to provide the data connectivity and propagation.

Unnecessary packet dissemination is obstructed by using density adaptive probability functions $p_{\text {same }}, p_{\text {opp }}$ and $p_{\text {sameToOpp }}$ which are the probabilities of forwarding packets in the same direction, in the opposite direction and transmitting packets from original direction to the opposite direction, respectively. This approach solves the broadcast storm problem by decreasing the collision rate. We have information about neighbors of each node by sending hello packets periodically. The neighbor number provides a simple but effective density adaptation in the algorithm.

In our system, there exist two types of packets. One of them is the periodic hello packet that includes source id, $\mathrm{x}-\mathrm{y}$ coordinates of the source and its traveling direction. When hello packets are received, the receiver node creates its neighborhood list which includes information about its neighbors. Hello packets are like control packets. By using this method, we can easily manipulate the number of neighbors and their locations. The second type is data packet which 
includes the senders' id, $\mathrm{x}-\mathrm{y}$ coordinates, the packet dissemination distance, the direction flag and the original flag. The dissemination distance shows how far the data packet should be propagated.

Algorithm 1 shows the decision phases of flag values in EpiDOL. While the vehicle is moving in the packets' dissemination direction (Line 1-2), if there are any same directional neighbors (Line 3 ), it means that packet will propagate in the original side, of is 1 (Line 4). Additionally, for providing the continuity of the packet dissemination, EpiDOL sends this packet to the opposite directional vehicles (Line 5) by setting the of and $d f$ to 0 (Line 6). Another case is when the value of $d f$ is 0 (Line 9-10). This shows that vehicle is moving in the opposite direction of the packets' dissemination. If there are any same directional neighbors (Line 11), packet is sending to them with the same flag values (Line 12). If there are any opposite directional neighbors (Line 13), which are actually in the original side, of value is set to 1 (Line 14), showing that the packet returns its original directional side.

\subsection{Probability Functions}

The decision of forwarding a packet or not is taken by a probabilistic manner at each node independently. Prior to the each packet transmission, a probability that estimates the necessity of the transmission of a packet from a particular node is calculated with the help of the number of total neighbors. We assumed that the more the neighborhood number is, the higher the chance of nodes receiving the data packet. With this assumption in mind the most trivial probability function is $p=1 / N$, where $\mathrm{N}$ is the number of neighbors, however it can easily be proven that this function will not perform good in dense networks. Within a neighborhood with $N$ nodes, the probability of a packet which is not transmitted by a particular node is $p^{c}=1-1 / N$. In homogeneously distributed dense network, we can safely assume that there are no clustering, so each node will approximately have the same number of neighbors. Since each node decides independently with the assumption of a packet is received by $\mathrm{N}$ nodes the probability of a packet not forwarded by any nodes is;

Let $N=\#$ of Neighbors. Then,

$$
p_{N}^{c}=\left(1-\frac{1}{N}\right)^{N}
$$

If we take the limit of this probability as $N$ goes to infinity, to see the probability of a packet being not forwarded by any nodes in a dense network,

$$
\begin{aligned}
\lim _{N \rightarrow \infty} p_{N}^{c} & =\lim _{N \rightarrow \infty}\left(1-\frac{1}{N}\right)^{N} \\
& =\lim _{N \rightarrow \infty}\left(\frac{1}{e}-\frac{1}{2 e N}-\frac{5}{24 e N^{2}}+\ldots\right)=\frac{1}{e} \approx 0.37
\end{aligned}
$$

This shows that, in dense networks since $p$ is so small, the dissemination of the packet will be stopped with approximately 0.37 probability. In real life, due 
to collisions in a dense network, the number of nodes that receive a packet is much less than N. Consequently the probability of a packet not forwarded by any nodes is even higher than 0.37 . For avoiding these situations, we multiply our value with $\alpha$ parameter. So we choose $p=\frac{\alpha}{N}$ which decreases the $p_{N}^{c}$ to $\frac{1}{e^{\alpha}}$ for large $N$. Note that the case of $p>1$ is treated as if $p=1$. Both $p_{\text {same }}$ and $p_{\text {opp }}$ are calculated with this function. However, according to our simulations, we detected that the best $\alpha$ values are different for propagating packets in the original and in the opposite directions. Thereon we use 2 different $\alpha$ values; $\alpha_{\text {same }}$ and $\alpha_{\text {opposite }}$ which are optimized by evaluating the different $\alpha$ values on various scenarios.

The decision of using opposite lane nodes as relay nodes not only depends on the number of neighbors but also depends on the spatial distribution of the nodes around. The extreme case is the vehicles at the rear end of a cluster. Basically they will have large number of neighbors due to number of vehicles that lead them, however these neighbors are not helpful for propagating packets to backward direction. With this intuition we proposed the following Algorithm 2 to decide on usage of opposite lane nodes. Therefore, the corresponding $p_{\text {sameToOpp }}$ is equivalent to $P\{\#$ BackwardNeighbors $\leq$ backwardValue $\}$. This function helps to solve the disconnected networks problem by continuing the packet dissemination using opposite sided vehicles as relaying nodes. Backward functions use Algorithm 2. In this function, only vehicles in the rear end of a connected cluster are sending packets to the opposite lane.

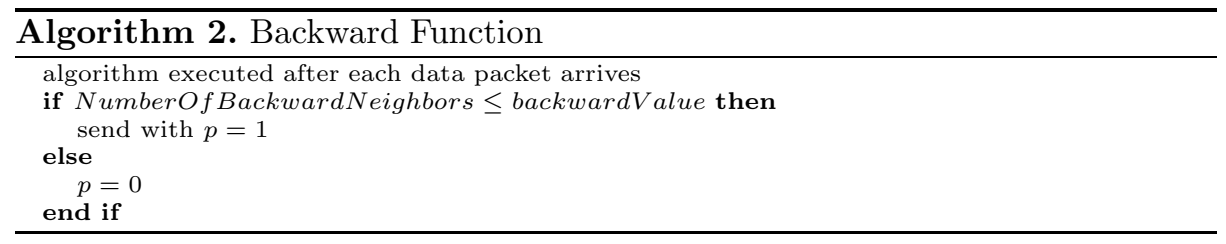

\section{Implementation}

In our simulations, we use SUMO [18 for generating realistic low and high density traces. The simulation environment is ns-3 [19]. According to [20], the typical transmission range is 400 meters. We consider a network with randomly distributed vehicles over a 6-lane bidirectional highway. Our region of interest (ROI) has a length of $5 \mathrm{~km}$. Since we assumed that there are not any intersections in our ROI, neither death nor birth of a node are allowed. To imitate the dynamic behavior of a highway, the speeds of the vehicles are uniformly distributed from $80 \mathrm{~km} / \mathrm{h}$ to $120 \mathrm{~km} / \mathrm{h}$.

\subsection{Performance Metrics}

EpiDOL is designed for merging epidemic approach with highly mobile ad-hoc networks. In order to evaluate its reliability and efficiency, we use the following performance metrics versus node density which is vehicles/square meter. Moreover, these metrics also enable an objective comparison of our proposed algorithm with other approaches. 
- End-to-End Delay: Time taken for packet transmission from source to nodes which are in the range of dissemination distance. For each packet received by every node, it is given by; End-to-End Delay $=t_{\text {receive }}-t_{\text {firstSending }}$.

- Throughput: This parameter shows the rate of successfully received packets by all nodes which are in the dissemination distance. Calculation is as follows: Throughput $=\frac{\# \text { received packets for each node }}{\text { \# all transmitted packets }} \times 100$.

- Opposite Lane: This parameter measures how many times opposite lane nodes resend the packets that are taken from the original side. Calculation is as follows: Opposite Lane $=\frac{\text { \#packets sent by opposite directional nodes }}{\text { \# all packets }} \times 100$.

- Overhead: The number of duplicate packets received during the simulation. The overhead is simply equal to the number of received duplicate packets at each node.

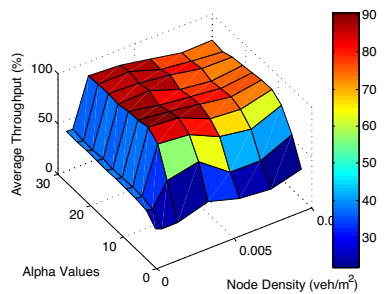

Throughput

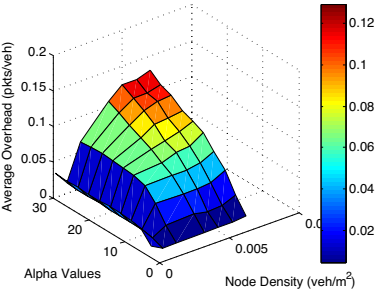

Overhead

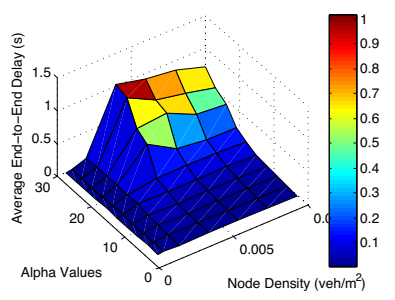

End-to-end Delay

Fig. 1. Choosing optimal $\alpha_{\text {same }}$

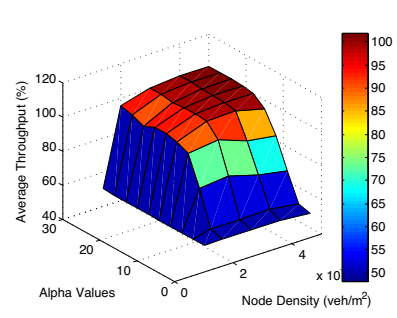

Throughput

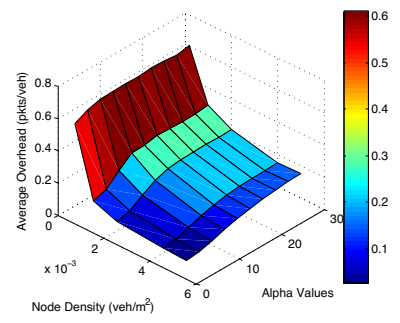

Overhead

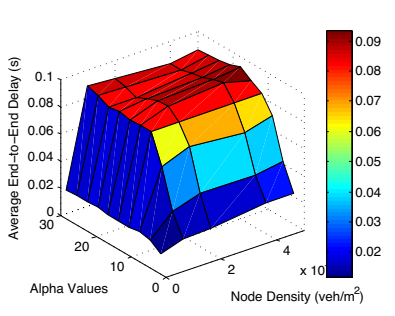

End-to-end Delay

Fig. 2. Choosing optimal $\alpha_{\text {opposite }}$

\subsection{Optimization of $\alpha_{\text {same }}$}

For optimization of the $\alpha_{\text {same }}$ value, we generated SUMO traces which include 10 to 500 vehicles ride in the same direction. Changing $\alpha$ values from 3 to the 30, we produced 3D graphs in Fig. 1. According to these results, $\alpha_{\text {same }}$ is chosen as 15 , since $90 \%$ throughput is achieved while the end-to-end delay is less than $0.06 \mathrm{~s}$ and the overhead is lower than 0.07 . $\alpha_{\text {same }}$ being equal to 15 ensures if we have less than 15 vehicles within the coverage area, all nodes will try to forward packets. This is desirable in a sparse network, since at low density our main concern is the survival of the packet rather than the packet collisions. Besides, as the neighbor numbers increase and $p_{\text {same }}$ decreases the expected number of 
retransmissions in a certain area will remain around 15. As seen in Fig. 1b, after a certain node density the overhead does not increase at all for $\alpha_{\text {same }}=15$. This supports our claim about our $p_{\text {same }}$ being sensitive to node density in the network. It is obvious that the performance can be increased with the perfect knowledge of the network density. However, due to excessive control packets, acquiring this information will increase the network overhead significantly, that might even decrease the overall throughput.

\subsection{Optimization of $\alpha_{\text {opposite }}$}

For optimization of the $\alpha_{\text {opposite }}$ value, we generated SUMO traces which include 10 to 300 vehicles in the opposite direction and 60 vehicles in the original (same) direction. For $\alpha$ values from 3 to 30, the results are shown in Fig. 2, According to these results, $\alpha_{\text {opposite }}$ is chosen as 21 , since $97 \%$ throughput is achieved while end-to-end delay is less than $0.1 \mathrm{~s}$ and overhead is lower than 0.1 . The optimal $\alpha_{\text {opposite }}$ being more than $\alpha_{\text {same }}$ is reasonable since we need more persistent transmissions to carry packets in between disconnected networks. Also Fig. 2a shows that even in really low densities, the utilization of the nodes in the opposite direction can double the throughput with $\alpha_{\text {opposite }}$ equals to 21 . This proves that the regardless of the number of nodes in the opposite direction, we should use them as relay nodes.

\subsection{Optimization of backwardValue}

backwardValue is used as a threshold in calculation of the $p_{\text {sameToOpp }}$ which decides whether sending packets from the original side to the opposite side or not. Consequently, choosing the best backwardValue is crucial for the performance of the algorithm. To reason about and select the optimal backwardValue, we simulated networks with different densities using backwardValue's from 0 to 17. Fig. 3 compares the throughput rates with different backwardValue's. However we should focus the part where node density is less than $3 \times 10^{-3}$. At higher densities, opposite lane usage does not really improve the throughput, since we do not observe disconnected networks problem anymore, consequently all backwardValue's converge to same throughput levels. However, the average overhead for densities higher than $3 \times 10^{-3}$ does not significantly differ for different backwardValue's as shown in Fig. 4. At high density conditions, the necessity of using opposite lane decreases. According to our function, the number of eligible vehicles which send packets to opposite lane also decreases while the density increases. In summary, by using the appropriate backwardValue we can double the throughput in low densities in return of higher overhead, however our main concern is to maintain connectivity rather than overhead in low densities. On top of this for higher densities, even though backward function can not improve the throughput, it also does not significantly increase the overhead which is the limiting factor. This proves the density adaptivity of our approach. To decide the optimal value of backwardValue, we have to consider Fig. 3 and Fig. 
4 simultaneously. According to Fig. 3, to achieve 90\% throughput in lower densities, backwardValue should be higher than 9. However, for backwardValue's greater than 9, the throughput does not increase at all. Furthermore, considering overhead values of Fig. 4 for several different vehicle densities, the optimum backwardValue is determined as 11. As shown in Fig. 5. an evaluation of this backwardV alue along with two others has been performed and these results indicate the density adaptive nature of opposite lane usage ratio with the probability function making use of number of backward neighbors.
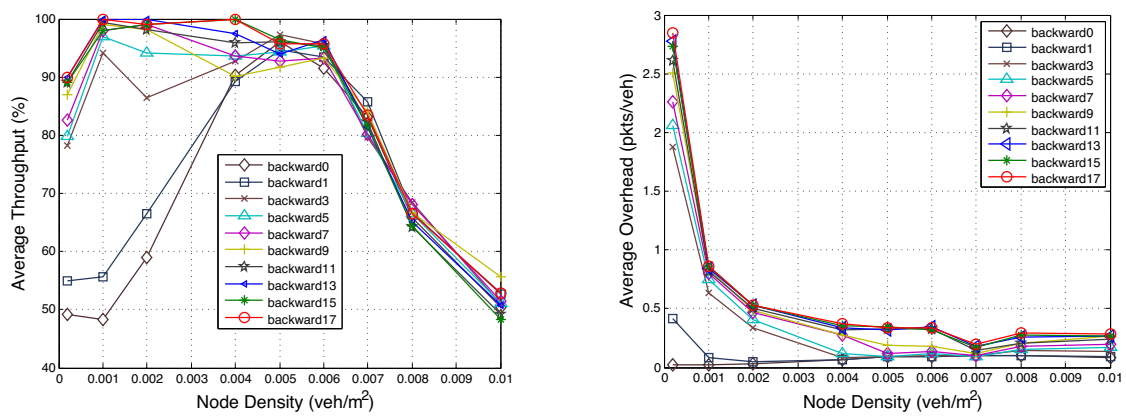

Fig. 3. Throughput for Backward Func- Fig. 4. Overhead for Backward Functions tions

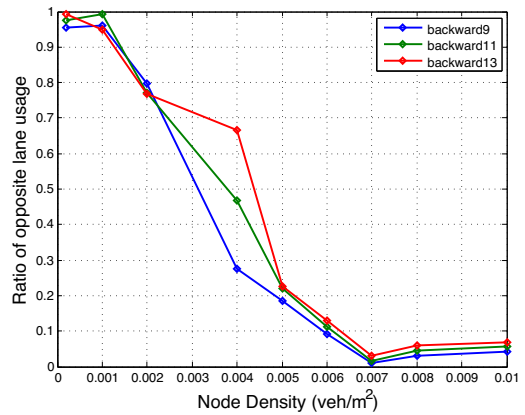

Fig. 5. Opposite Lane Usage Ratio

\subsection{Compared Protocols}

To prove the efficiency of EpiDOL, we compared it with state of the art data dissemination protocols designed for VANET, namely Edge-Aware 3 and DVCAST [4]. Edge-Aware is also an epidemic protocol that utilizes the GPS information. Basically it calculates the probability of rebroadcasting $P$ as

$$
P= \begin{cases}1, & \text { if } N_{f} \text { or } N_{b}=0 \\ 1-\exp \left(-\alpha \frac{\left|N_{f}-N_{b}\right|}{N_{f}+N_{b}}\right), & \text { otherwise }\end{cases}
$$

where $N_{f}$ and $N_{b}$ are the number of times the car has received that particular message from front and from back respectively. With this approach, they have managed to give higher probabilities to vehicles near the head or tail of a cluster. 
However, they have not proposed a specialized function to use opposite lane that can increase the connectivity and the throughput considerably for disconnected networks as we proved in this work. Also, the proposed protocol is only compared with simple flooding protocol in a controlled scenario rather than realistic traces.

DV-CAST [4 is a distributed broadcast protocol that utilizes opposite lane vehicles. By exchanging GPS information, every vehicle classifies its network as a well connected, sparsely connected or totally disconnected network. Then depending on this classification, vehicles set the values for three flags, MDC (message direction connectivity), DFlg (direction flag) and ODC (opposite direction connectivity). For different combinations of these flags, DV-CAST takes different actions such as broadcast suppression, rebroadcast, packet relaying, carry and forward and wait and forward. However, the simulations of this protocol is only limited with controlled circular highway scenario that lacks realistic traces such as SUMO traces.

Compared to these two protocols, in EpiDOL we use not only epidemic approach but also add some intelligence by making system robust to the density changes. To achieve an objective comparison, we use the metrics defined in Section 5 .

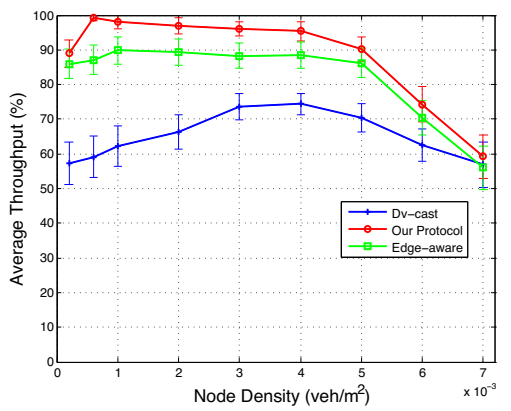

Fig. 6. Comparison of throughputs

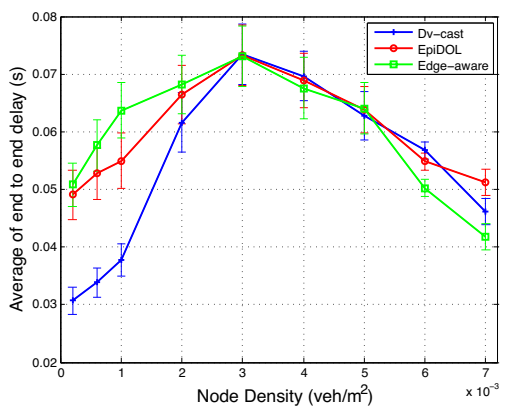

Fig. 8. Comparison of end-to-end de- Fig. 9. Comparison of opposite lane lays

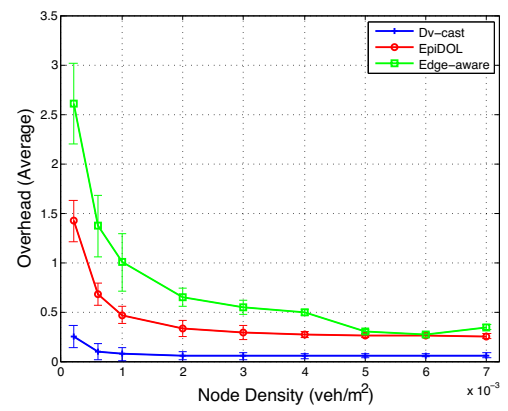

Fig. 7. Comparison of overheads

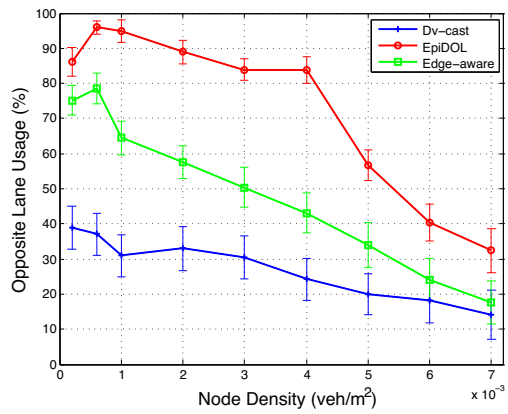
usage 


\section{Results}

We have compared the selected protocols in ns-3 with realistic SUMO traces by using 10 random runs. Our results are shown in Figures, 6,

Fig. 6] shows the ratio of successfully received packets by all same directional nodes in terms of percentage. Error bars in the figure show standard deviations around averages. In low densities, only EpiDOL achieves more than 90\% throughput. Higher opposite lane usage shown in Fig. 9 ensures higher throughput. This proves that our protocol handles the disconnected network problem more effectively than the other protocols. According to Fig. 8, end-to-end delays of all protocols are comparable. Since DV-CAST can not distribute packets to the whole network, it is expected to see lower end-to-end delays. Like Overhead of EpiDOL is lower than Edge-aware but higher than DV-CAST as shown in Fig. 7. However, in these densities our main concern is maintaining connection between disconnected networks rather than overhead.

In high densities, as seen in Fig. 6 throughput is slightly better than the other protocols. Fig. 7 shows that this improvement is realized without introducing additional overhead to the network. EpiDOL has managed to deliver packets to higher number of nodes with comparable end-to-end delays as seen in Fig. 8 . Achieving higher throughputs with low overheads in high densities shows that EpiDOL was able to deal with broadcast storm problem.

\section{Conclusion}

We proposed EpiDOL which is an epidemic and density adaptive protocol for data dissemination in VANETs that utilizes the opposite lane capacity with novel probability functions. We have optimized parameters of the algorithm based on analysis by using traffic traces from SUMO. We compared the performance of the proposed algorithm with the existing algorithms in terms of end-to-end delay, overhead, throughput and opposite lane usage.

According to results in low densities we achieved more than the $90 \%$ throughput with comparable end-to-end delay, overhead and opposite lane usage. This showed that EpiDOL handled the disconnected network problem. In high densities, without excessive values in end-to-end delay and overhead, throughput achieved by EpiDOL is better than the others. This also indicates that broadcast storm problem did not effect our protocol due to its probabilistic density adaptive functions.

As a future work, we aim to focus on the analysis of the performance of EpiDOL for different data dissemination scenarios.

Acknowledgement. This work has been conducted as part of Turk Telekom Research project under Grant Number 11315-07.

\section{References}

1. Biswas, S., Tatchikou, R., Dion, F.: Vehicle-to-vehicle wireless communication protocols for enhancing highway traffic safety. IEEE Communications Magazine 44(1), 74-82 (2006) 
2. Li, F., Wang, Y.: Routing in vehicular ad hoc networks: A survey. Vehicular Technology Magazine 2(2), 12-22 (2007)

3. Nekovee, M.: Epidemic algorithms for reliable and efficient information dissemination in vehicular. Intelligent Transport Systems, IET 3(2), 104-110 (2009)

4. Tonguz, O., Wisitpongphan, N., Bai, F.: Dv-cast: A distributed vehicular broad- cast protocol for vehicular ad hoc networks. IEEE Wireless Communications 17(2), 47-57 (2010)

5. Fleetnet project (2003), http://www.neclab.eu/Projects/eetnet.htm

6. Cartel project, http://cartel.csail.mit.edu/doku.php

7. Tonguz, O., Wisitpongphan, N., Bai, F., Mudalige, P., Sadekar, V.: Broadcasting in vanet. In: 2007 Mobile Networking for Vehicular Environments, pp. 7-12 (May 2007)

8. Wisitpongphan, N., Tonguz, O., Parikh, J., Mudalige, P., Bai, F., Sadekar, V.: Broadcast storm mitigation techniques in vehicular ad hoc networks. IEEE Wireless Communications 14(6), 84-94 (2007)

9. Schwartz, R., Ohazulike, A., Sommer, C., Scholten, H., Dressler, F., Havinga, P.: Fair and adaptive data dissemination for traffic information systems. In: 2012 IEEE Vehicular Networking Conference (VNC), pp. 1-8 (2012)

10. Blum, J., Eskandarian, A., Hoffman, L.: Challenges of intervehicle ad hoc networks. IEEE Transactions on Intelligent Transportation Systems 5(4), 347-351 (2004)

11. Taleb, T., Ochi, M., Jamalipour, A., Kato, N., Nemoto, Y.: An efficient vehicleheading based routing protocol for vanet networks. In: Wireless Communications and Networking Conference, WCNC 2006, vol. 4, pp. 2199-2204. IEEE (April 2006)

12. Taleb, T., Sakhaee, E., Jamalipour, A., Hashimoto, K., Kato, N., Nemoto, Y.: A stable routing protocol to support its services in vanet networks. IEEE Transactions on Vehicular Technology 56(6), 3337-3347 (2007)

13. Costa, P., Gavidia, D., Koldehofe, B., Miranda, H., Musolesi, M., Riva, O.: When cars start gossiping. In: Proceedings of the 6th Workshop on Middleware for Network Eccentric and Mobile Applications, MiNEMA 2008, pp. 1-4 (2008)

14. Meireles, R., Steenkiste, P., Barros, J.: Dazl: Density-aware zone-based packet forwarding in vehicular networks. In: 2012 IEEE Vehicular Networking Conference (VNC), pp. 234-241 (2012)

15. Naumov, V., Baumann, R., Gross, T.: An evaluation of inter-vehicle ad hoc networks based on realistic vehicular traces. In: Proceedings of the 7th ACM International Symposium on Mobile Ad Hoc Networking and Computing, MobiHoc 2006, pp. 108-119 (2006)

16. Zuo, J., Wang, Y., Liu, Y., Zhang, Y.: Performance evaluation of routing protocol in vanet with vehicle-node density. In: 2010 6th International Conference on Wireless Communications Networking and Mobile Computing (WiCOM), pp. 1-4 (September 2010)

17. Ieee standard for information technology- local and metropolitan area networks specific requirements- part 11: Wireless lan medium access control (mac) and physical layer (phy) specifications amendment 6: Wireless access in vehicular environments, IEEE Std 802.11p-2010 (Amendment to IEEE Std 802.11-2007 as amended by IEEE Std 802.11k-2008, IEEE Std 802.11r-2008, IEEE Std 802.11y- 2008, IEEE Std 802.11n-2009, and IEEE Std 802.11w-2009), pp. 1-51, 15

18. Sumo - simulation of urban mobility, http://sumo.sourceforge.net

19. Network simulator, ns-3, http://www.nsnam.org/

20. Zhang, M., Wolff, R.: Routing protocols for vehicular ad hoc networks in rural areas. IEEE Communications Magazine 46(11), 126-131 (2008) 